\title{
Frame Synchronization for the DTMB Systems with the Multiple-antenna Receivers
}

\author{
Zi-Wei Zheng ${ }^{1,2}$ \\ ${ }^{1}$ College of Information Science and Engineering, Ningbo University, 315211 Ningbo, P. R. China \\ ${ }^{2}$ College of Information Engineering, Dalian Maritime University, 116026 Dalian, P. R. China \\ E-mail: ziwei_zheng@yahoo.com.cn
}

Keywords: Frame synchronization; DTMB systems; multiple-antenna receivers.

\begin{abstract}
Faced with the challenges of modern communication, high data throughput requirements, extensive research has been paid to broadband modulation. The multi-carrier modulation is one of the most important wide band modulation techniques. Orthogonal frequency division multiplexing (OFDM) is an effective blow to the broadcast channel multipath fading and high bit rate transmission multi-carrier modulation technology of wireless channels, it is widely used in modern digital television terrestrial broadcasting (DTTB) system to support performance bandwidth-efficient multimedia services with the aid of inverse fast Fourier transform and fast Fourier transform operation. Multiple-antenna transmitter and receiver technology allows several times to achieve data transmission rates and spectrum efficiency and the use of multiple transmit antennas and multiple receive antennas through spatial processing. The Digital Terrestrial Multimedia Broadcasting (DTMB) system with the multiple-antenna receiver is considered in this paper. Digital Terrestrial Multimedia Broadcasting (DTMB) has three kinds of Frame Head mode with PN420/PN595/PN945 as training sequences for the time domain synchronous OFDM (TDS-OFDM). At the receiver side with multiple antennas, Frame Head mode detection should be done. In this paper, the frame synchronization scheme is proposed for the DTMB systems with the multiple-antenna receiver. System performance studies under different channel situations are used to verify the efficiency of the proposed scheme for the DTMB systems with the multiple-antenna receiver.
\end{abstract}

\section{Introduction}

Faced with the challenges of modern communication, high data throughput requirements, extensive research has been paid to broadband modulation. The multi-carrier modulation is one of the most important wide band modulation techniques. Orthogonal frequency division multiplexing (OFDM) is an effective multi-carrier modulation technique for combating radio channel multipath fading and for high-bit-rate transmission over wireless channels with the aid of inverse fast Fourier transform and fast Fourier transform operations and it is extensively used in modern digital television terrestrial broadcasting (DTTB) systems to support high performance bandwidth-efficient multimedia services, such as the Digital Video Broadcasting for Terrestrial Television (DVB-T) proposed as the European digital television standard [2], and the Digital Terrestrial Multimedia Broadcasting (DTMB) [3] proposed as the digital television terrestrial broadcasting (DTTB) standard of the People's Republic of China.

When the length of the delay spread is greater than the guard interval, it significantly reduce the performance of the OFDM system [1]. Long guard interval in order to cope with multipath propagation in digital terrestrial television broadcasting, may introduce a lower cost effective data transfer rate, or the long OFDM symbols can use the saved data transfer rate, but in the implementation of the complexity and long-term cost of decoding delay. Therefore, the length of the guard interval of the OFDM symbol length must be trade-offs between system performance and the actual implementation of the complexity. 
The Signal Frame in the DVB-T standard consists of two parts, the Frame Head (the guard interval) and the Frame Body. The cyclic prefix (CP) is used as the Frame Head (the guard interval) and for coarse symbol synchronization to cope with multipath signals. Furthermore, DVB-T sends a large amount of training symbols (more than $10 \%$ of the data symbols) in order to facilitate the fine symbol synchronization and channel estimation [2].

In Chinese OFDM-based DTTB systems, instead of the cyclic prefix, time domain synchronous OFDM (TDS-OFDM) inserts pseudo-random number (PN) sequence as the guard interval, which also serves for synchronization and channel estimation [3]. After removing the PN sequence at the receiver, TDS-OFDM is equivalent to the zero-padded OFDM (ZP-OFDM) [4]. In [4], Muquet et al. proposed a low complexity equalizer for ZP-OFDM, compared ZP-OFDM and CP-OFDM, and numerically showed that ZP-OFDM with the equalizer outperformed CP-OFDM in terms of bit error rate, both in coded and un-coded cases. The reasonable time domain combination of the guard interval and the training symbol reduces transmission overhead and provides a better channel spectral efficiency performance. The separation of training and data makes the synchronization independent on the specific modulation type for the useful data and increases the modularity of the entire system. The Signal Frame in Chinese digital television terrestrial broadcasting standard consists of two parts, the Frame Head (the guard interval) and the Frame Body. The technique of multiple antennas at the transmitter and at the receiver permits several fold increase in achieved data rates and spectral efficiency through spatial processing and the use of multiple transmit antennas and multiple receive antennas. The Chinese DTTB system with multiple-antenna receiver is considered. There are three kinds of Frame Header, with PN420/PN595/PN945. The Frame Body processing block has two options, one is the single carrier modulation scheme, and the other is the OFDM-based multi-carrier modulation scheme. In this paper, we consider the situation that three kinds of Frame Head modes with PN420/PN595/PN945 as training sequences are used for the TDS-OFDM-based DTTB systems. At receiver side, Frame Head mode detection is important and should be done before subsequent synchronization and channel estimation.

\section{Problem Representation}

The Signal Frame in Chinese digital television terrestrial broadcasting standard consists of two parts, the Frame Head and the Frame Body, which is shown in figure 1. There are three kinds of Frame Header modes, with PN420 sequence, PN595 sequence, or PN945 sequence. The Frame Body processing block has two options, one is the single carrier modulation scheme, and the other is the OFDM-based multi-carrier modulation scheme. In this paper, we consider the situation that three kinds of Frame Head modes with PN420/PN595/PN945 as training sequences are used for the TDS-OFDM-based DTTB systems. PN420 sequence and PN945 sequence are made up of complete M-sequences of length 255 and length 511, and with their respective cyclical extensions as pre-ambles and post-ambles. PN595 sequence is made up of the first 595 symbols of an M-sequence of length 1023 and it has no cyclical characteristics. The initial phase of the generation polynomial is fixed for PN595 sequence. The initial phase of the generation polynomial can be optionally rotated within each super-frame for PN420 sequence and PN945 sequence. In this paper, we also simply assume that the initial phase of the generation polynomial is fixed for PN420 sequence and PN945 sequence. The power of the Frame Head is also different between PN595 sequence and the other two PN420 sequence, PN945 sequence. The power of the Frame Head PN595 sequence has the same average power as the Frame Body. The power of the Frame Head sequence PN420 sequence or PN945 sequence has an average power that is $3 \mathrm{~dB}$ higher than that of the Frame Body. The length of pre-amble ${ }^{L_{\text {pre }}}$ is 82 and the post-amble ${ }^{L_{\text {post }}}$ is 83 for the PN420 Frame Head mode. The length of pre-amble ${ }^{L_{p r e}}$ is 217 and the post-amble ${ }^{L_{p o s t}}$ is 217 for the PN945 Frame Head mode. Similarly, we

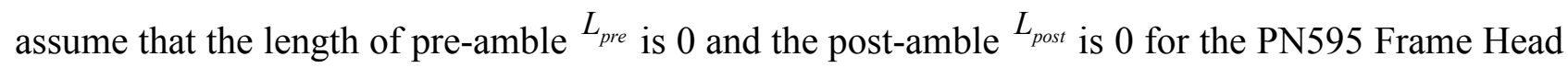


mode. BPSK modulation is used in the Frame Head for robust synchronization and channel estimation. The Frame Body has 3780 symbols, 3744 symbols carry the data and 36 symbols carry the Transmission Parameter Signaling (TPS). In this paper, we only consider the situation that OFDM modulation scheme is used for Frame Body. The TPSs carry information for the demodulator to automatically adapt to the incoming transmission such as FEC inner code rate, time interleaver length etc. The sub-carrier position and the symbol information of part of the TPSs are known at the receiver.

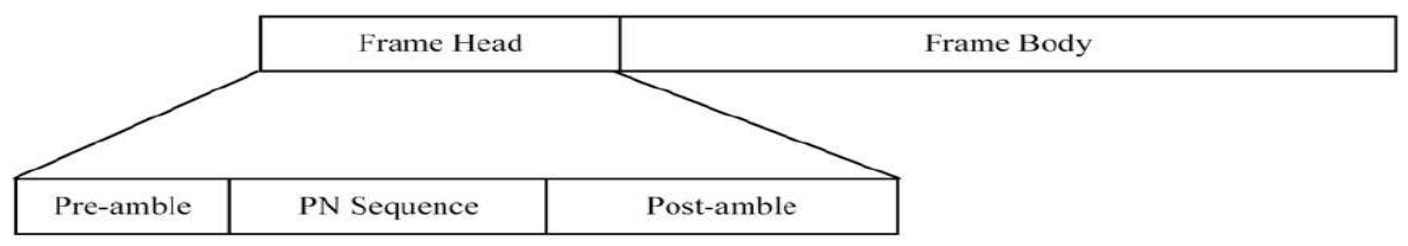

Fig. 1. Signal frame format for the DTMB systems with the multiple-antenna receiver

At the transmitter side of Chinese TDS-OFDM-based DTTB system, each 3780 of the mapping symbols are grouped into one OFDM frame body. Information symbols $\mathbf{s}(k)=\left[s_{0}(k), s_{1}(k), \cdots, s_{N_{s}-1}(k)\right]^{T}$ $\left(N_{s}=3780\right)$ are assigned to every specific OFDM data frame body by the inverse fast Fourier transform (IFFT) block. PN sequence $\mathbf{t}(k)=\left[t_{0}(k), t_{1}(k), \cdots, t_{N_{t}-1}(k)\right]^{T}$, is inserted for Frame Head mode detection, synchronization and channel distortion evaluation in front of each OFDM frame body. A square root raised cosine (SRRC) pulse shaping is performed to limit the transmitted signals to $8 \mathrm{MHz}$ bandwidth before they are up-converted and power amplified and transmitted over the channel. The symbol rate is same for both the Frame Head and the Frame Body, which is 7.56 MSPS.

At the multiple-antenna receiver side of Chinese TDS-OFDM-based DTTB system, the multiple-antenna received signals are down-converted, SRRC pulse shaping, sampled. Then, the samples are filling in the buffer, and then fed in parallel to the Frame Head mode detector, the timing estimator, the frequency offset estimator, the channel estimator, and the fast Fourier transform (FFT) block, respectively. Every kind of PN sequence (PN255 sequence for PN420 Frame Header mode, PN511 sequence for Frame Header PN945 mode, PN595 sequence for PN595 Frame Header mode), the same as the transmitted Frame Header, is generated by the local PN code generator and saved in the register. With the local generated PN sequences, the Frame Head mode detection, the timing and the frequency offset estimation are performed. In addition, the local generated PN sequences are also used as the channel-sounding signal. Timing synchronization is used to detect the symbol boundary and to compensate the sampling clock offset caused by clock frequency mismatch between receiver and transmitter. Carrier frequency synchronization is used to compensate the carrier frequency offset caused by carrier frequency mismatch between the transmitter and the receiver.

For the PN420 mode in the Chinese TDS-OFDM-based DTTB system, the initial phase of PN sequence is changed frame by frame. Although the initial phase of PN sequence is changed for the PN420 mode, there exists an identical PN255 sequence in all the signal frame headers with different pre-amble and post-amble. For the PN945 mode in the Chinese TDS-OFDM-based DTTB system, the initial phase of PN sequence is changed frame by frame. Although the initial phase of PN sequence is changed for PN945 mode, there exists an identical PN511 sequence in all the signal frame headers with different pre-amble and post-amble. For PN595 mode, since there is no pre-amble and post-amble in the Frame Header and the Frame Header is an identical PN595 sequence. In a multipath channel, when the transmitted PN sequence is aligned with the receiver identical PN sequence, correlation peaks can be observed depending on the span (measured in signal sampling period) of multipath delay profile. Because of its delta-like auto-correlation property, the different length and the different makeup for the unique PN sequence, respectively, in the PN420 Frame Header mode, the PN945 Frame Header mode, the PN595 Frame Header mode allow us to find the precise Frame Head mode and the timing position (the OFDM symbol boundary). Let $r(n)$ denote the sampled received signal at time-instant $n$ and let $a$ denote the slip between the received PN sequence and the locally 
generated identical PN sequence $P N$ of the tested mode. Here this slip is assumed to be an integer. The way to detect the Frame Head mode and to select the first channel tap in order to accurately locating the OFDM symbol boundary is proposed in this paper. The Frame Head mode detection and the OFDM symbol boundary detection metrics are defined as follows for the DTMB systems:

$$
\Lambda_{a_{r 1}}(n, a)=\frac{\left|\sum_{i=1}^{M} \sum_{a_{r}=1}^{a_{R}} r_{a_{r}}\left(n-i-L_{p o s t}\right) P N^{*}(n-i-a)\right|^{2}}{\left(\sum_{i=1}^{M} \sum_{a_{r}=1}^{a_{R}}\left|r_{a_{r}}\left(n-i-L_{p o s t}\right) P N^{*}(n-i-a)\right|\right)^{2}}
$$

and

$$
\Lambda_{a_{r 2}}(n)=\frac{r_{a_{\text {aver }}}(n)}{r_{a_{r \text { aver }}}(n-1)}
$$

where

$$
r_{a_{\text {raver }}}(n)=\frac{1}{K_{S}} \sum_{k=0}^{K_{S}-1 a_{r}=1} \sum_{R}\left[\left|r_{a_{r}}\left(n-K_{S}+1+k\right)-r_{a_{r}}\left(n-K_{S}+1+k-N_{s}-N_{t}\right)\right|^{2}\right]
$$

The PN sequence is with $M$ samples length of the tested Frame Header mode $(M=255, M=595$, or $M=511)$, it is assumed that there are $a_{R}$ antennas at the receiver. The length of post-amble ${ }^{L_{p o s t}}$ is $L_{p o s t}=83, L_{p o s t}=0, L_{p o s t}=217$, the length of PN sequence $N_{t}$ is $N_{t}=420, N_{t}=595, N_{t}=945$, respectively, for the tested PN420 Frame Header mode (the identical PN sequence is with $M=255$ samples length), the tested PN595 Frame Header mode (the identical PN sequence is with $M=595$ samples length), the tested PN945 Frame Header mode (the identical PN sequence is with $M=511$ samples length). $K_{S}$ is a small value for sampled averaging. For the Chinese TDS-OFDM-based DTTB systems, we let $K_{S}=10$

During the Frame Head, $\Lambda_{a_{r 2}}(n)$ is a value approximated to zero. However, when $n$ is at the starting point of the OFDM Frame Body, the value of $\Lambda_{a_{r 2}}(n)$ becomes abruptly to a very larger value. If at this $n$ point (the starting point of the OFDM frame body), the value of $\Lambda_{a_{r 1}}(n, a)$ is larger than the predefined threshold $\chi$, the tested Frame Head mode detection and the OFDM symbol boundary location is achieved. To further improve the frame synchronization performance for the DTMB Systems with the Multiple-antenna Receiver, several frame are used and iteratively smoothed.

It is assumed that there are four antennas at the receiver, the simulation channels are the AWGN channel, the simulation channels are the DVB-T Fix Reception F1 version channel model [2], the 6-path typical hilly terrain channels with parameters randomly generated from COST 207 specifications [5] under different maximal Doppler frequencies, $f_{d}=10 \mathrm{~Hz}$, which corresponds to receiver velocity of 12.5 to $23 \mathrm{~km} / \mathrm{h}$ in the TV UHF band $(@ 470 \ldots 862 \mathrm{MHz})$, and $f_{d}=60 \mathrm{~Hz}$, which corresponds to receiver velocity of 75 to $138 \mathrm{~km} / \mathrm{h}$ in the TV UHF band (@470...862MHz). Local oscillator crystal offset normalized the sampling period is $10 \mathrm{ppm}$, carrier-frequency offset is of 3 -subcarrier spacing. In this paper, we also use $\chi_{1}=0.3$ and $S N R_{1}=10 \mathrm{~dB}$ as the references in other channel situations. The missed OFDM symbol boundary probability vs. false Frame Head mode detection probability performances are shown in figure 2 for the Chinese TDS-OFDM-based DTMB system under different channel situations. From figure 2, we can see that the proposed Frame Head mode detection and the OFDM symbol boundary detection scheme make the Chinese TDS-OFDM-based DTMB system with robust detection capability. 


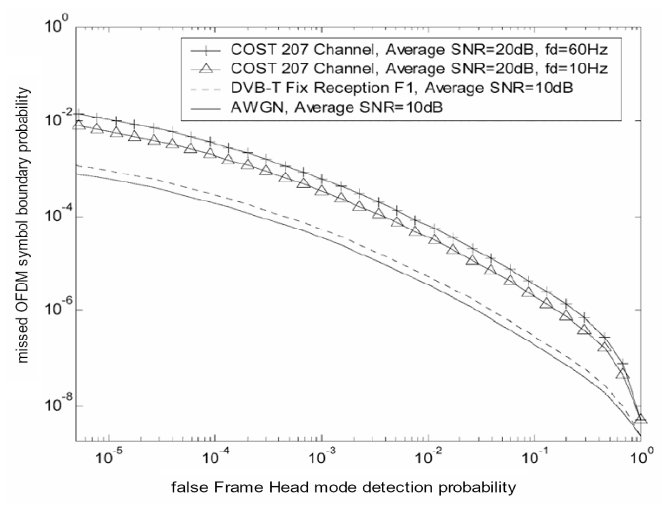

Fig. 2. Missed OFDM symbol boundary probability vs. false Frame Head mode detection probability under different channel situations

\section{Summary}

A frame synchronization scheme is proposed for the DTMB systems with the multiple-antenna Receiver. System performance studies under different channel situations are used to verify the efficiency of the proposed scheme.

\section{Acknowledgments}

This work was supported in part by the National Science Foundation of China (No. 60972063), the National Science and Technology Major Project (No. 2011ZX03002-004-02), the Science Foundation of Zhejiang Province (No. R1110416), the Specialized Research Fund for the Doctoral Program of Higher Education (No. 20113305110002), the Program for Technology Innovation Team of Ningbo Government (No. 2011B81002).

\section{References}

[1] R. V. Nee and R. Prasad, OFDM for wireless multimedia communications. Boston: Artech House, 2000.

[2] ETSI EN 300744 V1.4.1, Digital Video Broadcasting (DVB); Frame structure, channel coding and modulation for digital terrestrial television, Jan. 2001.

[3] GB 20600-2006, National Standard of P. R. China; Frame structure, channel coding and modulation for digital terrestrial television, Aug. 2006.

[4] B. Muquet, Z. Wang, G.B. Giannakis, M. de Courville, and P. Duhamel, "Cyclic-Prefixed or Zero-Padded Multicarrier Transmissions?," IEEE Trans. Commun., vol. 50, pp. 2136 -2148, Dec. 2002.

[5] M. Failli, "Digital land mobile radio communications," CIC Inf. Technol. and Sciences, Brussels, Belgium, COST 207 Final Rep., 1989, pp. 135-166. 